\title{
Herederas en el código civil: discursos y representaciones en torno a la capacidad jurídica de las mujeres*
}

\author{
Heiresses in the civil code: \\ discourses and representations \\ regarding legal capacity of women
}

\author{
Romina Soledad Coronello \\ Consejo Nacional de Investigaciones Científicas y Técnicas \\ Universidad Nacional de Mar del Plata \\ (Argentina) \\ rs_coronello@hotmail.com
}

\section{Resumen}

La preocupación por el lugar de la mujeres en los mecanismos de sucesión, ha sido abordada tangencialmente por estudios centrados en el siglo XIX, que analizan las redefiniciones jurídicas en torno a la familia, la propiedad y la herencia destacando cambios en la legislación familiar a partir de 1830. Por otro lado, los estudios de Dora Barrancos señalan la obturación de los derechos civiles de las mujeres y la consecuente inferiorizacion de la condición jurídica de la misma, sosteniendo la agudización de la subordinación legal femenina en el tránsito del siglo XVIII al XIX. Teniendo en cuenta estos aportes, nos proponemos comenzar a explorar las redefiniciones jurídicas del lugar de las mujeres en las dinámicas de sucesión del período. Para ello y como punto de partida de nuestra investigación, focalizaremos en este trabajo específicamente sobre las disposiciones del Código Civil de 1869 así como sobre las concepciones jurídicas y circunstancias que las explican en un intento por historizar las disposiciones en pos de esbozar cambios y continuidades, así como indagar sobre las representaciones que implican tales discursos.

Palabras Clave: Legislación - Herencia - Mujeres - Capacidad jurídica

\footnotetext{
* Agradezco a los evaluadores anónimos los comentarios, observaciones y sugerencias que me han llevado a realizar mejoras en la versión de este trabajo.
} 


\begin{abstract}
The concern about the place of women in the heritage dynamic has been incidentally tackled by studies that analyze the legal redefinitions about family, property, and heritage during the XIX century, foregrounding changes on family legislation since 1830. On the other hand, Dora Barrancos pointed that codification has worsened women's legal status and condition. Taking into account these contributions, we propose to explore the legal redefinitions of women's place in heritage dynamic in the transit from the XVIII to the XIX century. As a starting point of that investigation we will focus on this article about the Argentinean Civil Code from 1869 as well as the legal conceptions and the circumstances than explain its dispositions, trying to historicize it outlining changes and continuities. We will also inquire into the representations that those discourses implied.
\end{abstract}

Key Words: Legislation - Heritage - Women - Legal capacity

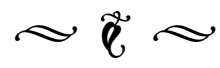

\section{Introducción}

La cuestión de la formación estatal ha cobrado nuevo impulso en las últimas décadas producto de miradas renovadas en la historiografía política que complejizaron las visiones tradicionales sobre la temática desde nuevos enfoques y problemas. De esta forma, la imagen unidireccional de un Estado abstracto y auto centrado que se impone sobre la sociedad civil fue comenzando a ser cuestionada y revisitada a la luz de estos nuevos abordajes. ${ }^{1}$ Los mismos implicaron, por un lado, el desplazamiento de la atención del centro a la periferia, así como, la modificación de las escalas de observación y el período, teniéndose en cuenta las formas de organización política previas a 1852 . Como resultado, han emergido imágenes

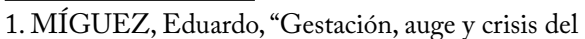
orden político oligárquico en la Argentina. Balance de la historiografía reciente", en Polhis, Buenos Aires, primer semestre 2012, Año 5, № 9, pp. 38-68. que resaltan la importancia de las relaciones interpersonales como base operativa del orden social donde se destaca el rol desempeñado por las elites provinciales en el proceso, así como, las formas negociadas que implicaban los vínculos entre poderes locales y poder central. Desde esta perspectiva, resulta más acertado plantear que más que ser producto de progresivas y simultáneas penetraciones en las provincias, el proceso de centralización del poder resultó tributario de dos dinámicas: la provincial y la nacional. Ello no implicó por lo tanto un proceso unilineal carente de conflictos sino que, por el contrario, demuestra que no hubo un camino necesario de tránsito hacia la conformación de un poder central consolidado, existiendo distintos ensayos institucionales que enfrentaron diversas dificultades: escasez de recursos económicos y humanos, así como, la dificultad de construir formas simbólicas impersonales de respeto a las instituciones en reemplazo de los sistemas de lealtades. ${ }^{2}$

2. BRAGONI, Beatriz y MÍGUEZ, Eduardo (coordinadores) Un nuevo orden político. Provincias y 
En síntesis, tales avances nos permiten comprender el proceso de formación del Estado como una forma de organización central que se creó a partir de la convergencia de, al menos, catorce formas que le precedieron. De esta forma, el énfasis más que en la progresiva adquisición de atributos propios del Estado moderno, se encuentra en los procesos de construcción de nuevas formas de autoridad política, vínculos sociales, instituciones. ${ }^{3}$ Dentro de esta perspectiva, creemos que es posible analizar la creación y consolidación del ordenamiento jurídico como parte del proceso de construcción del nuevo orden social, político e institucional.

Los estudios sobre ordenamientos jurídicos y más específicamente del Código Civil (el intento más claro de unificar y dar sentido al conjunto de normas existentes) han sido objeto de la historia del derecho ${ }^{4}$ de la primera mitad del siglo XX que, ligada a la historia de las instituciones, privilegiaba la descripción de los sistemas jurídicos distinguiendo aquellos de la etapa colonial de los de la poscolonial. Prestando menos atención a las continuidades doctrinarias, normativas y procedimentales, postuló el pasaje de un "derecho indiano" a otro "patrio" dentro del cual, el Código Civil marcaría un punto de inflexión en el camino de un proceso tendiente a la implementación de un principio de ley superador, inspirado en las

Estado Nacional, 1852-1880, Biblos, Buenos Aires, 2010, pp. 9-28.

3. FASANO, Juan Pablo, "Historia de la justicia e historia política. Apuntes para un diálogo", ponencia presentada en VIJornadas de Historia Politica. Argentina, siglos XIX y XX, UnSam, 2011.

4. DALLA CORTE, Gabriela, "La historia del derecho en la Argentina o la historia jurídica como proceso", en Prohistoria, Rosario, 1999, № 3, pp. 133-173. nuevas ideas jurídicas a partir de la influencia de modelos como la tradición napoleónica. ${ }^{5}$

Sin embargo, en las últimas décadas, se ha ido conformando en nuestro país una perspectiva historiográfica que partiendo desde distintas historias ${ }^{6}$ así como de la valorización de fuentes como las judiciales, ha renovado el enfoque desde el cual se analizan los fenómenos jurídicos atendiendo a la historicidad del derecho, es decir, al derecho como fenómeno social resultante de una construcción histórica. Esta mirada, producto del acercamiento entre historia social e historia jurídica, devino en el estudio de la justicia desde distintas aristas. Entre ellas, los estudios sobre la administración de la justicia iluminaron las relaciones entre ésta y el poder, así como los trabajos sobre los usos de la misma por parte de los distintos actores sociales revalorizaron las dimensiones no estatales de lo jurídico. ${ }^{7}$

5. BARRIERA, Darío, "Justicias, jueces y culturas jurídicas en el siglo XIX rioplatense", Nuevo Mundo Mundos Nuevos [En línea], Debates, 2010.Disponible en: <http://nuevomundo.revues.org/59252>; ZEBERIO, Blanca, "Los derechos civiles en la transición.

Controversias jurídicas y proyectos políticos (siglos XIXXX)", en BONAUDO, Marta, REGUERA, Andrea y ZEBERIO, Blanca (coordinadoras) Las escalas de la historia comparada. T. I: Dinámicas sociales, poderes políticos y sistemas jurídicos, Miño y Dávila editores, Buenos Aires, 2008, p. 286.

6. A partir de acercamientos analíticos provenientes de diversas vertientes históricas tales como la historia, social, cultural y política. Por otro lado, es interesante el comentario al respecto de Gabriela Tío Vallejo sobre la renovación de la historia jurídica como subproducto de las investigaciones sobre aquellos sectores que podían ser interpelados desde las fuentes judiciales.

7. Para un detallado recorrido al respecto consultar entre otros: BARRIERA, Darío y DALLA CORTE CABALLERO, Gabriela, "La ventana indiscreta. La Historia y las Antropología Jurídicas a través de las emociones de sus textos", Prohistoria, Rosario, 2001, Año V, No 5, 11-14. Disponible en: <http://es.scribd. 
La comprensión de las relaciones entre normas y prácticas sociales subyacen las indagaciones de estos diversos trabajos así como las preocupaciones que atañen a la construcción del poder político. En este sentido, frente a una tradición que enfatizaba las discontinuidades entre un orden jurídico legal colonial y el pos revolucionario, las nuevas formulaciones adoptan una perspectiva más atenta a las continuidades "afinando la mirada para analizar el problema de la construcción de un sistema político representativo y sus pasos hacia la formación de un poder judicial letrado e independiente."

Siguiendo los aportes planteados desde esta renovada historia jurídica, el derecho - así como el Estado - es también producto de una construcción histórica, y como tal no puede entenderse sin referirse a los elementos que se ponen en juego en su constitución: políticos, ideológicos, culturales. Es por ello que resulta necesario rastrear las razones que inspiran los cambios así como las permanencias, ya sean circunstancias políticas, sociales y económicas, de forma de explicar el contenido de las normas y eventualmente sus consecuencias prácticas.

Dentro de estas indagaciones, nos proponemos comenzar a explorar las

$\mathrm{com} / \mathrm{doc} / 27683247 / \mathrm{PROHISTORIA-05-2001-}$ COMPLETA>

BARRIERA, Darío, dossier "Justicias, jueces y culturas jurídicas en el siglo XIX rioplatense", en Nuevo Mundo Mundos Nuevos. Debate, 2010. Disponible en: <http:// nuevomundo.revues.org/59252»; TIO VALLEJO, Gabriela, "Los historiadores hacen justicia: un atajo hacia la sociedad y el poder en la campaña rioplatense en la primera mitad del siglo XIX", en Revista de Historia del Derecho, Buenos Aires, No 41, 2011.

8. BARRIERA, Gabriel, dossier “Justicias...", Op. Cit. 44 redefiniciones jurídicas del lugar de las mujeres en las dinámicas de sucesión.

Historiográficamente, si bien en las últimas décadas se han desarrollado trabajos que emprenden el análisis de los sistemas normativos decimonónicos adoptando una perspectiva de género, ${ }^{9}$ los estudios que abordan el lugar de las mujeres en los mecanismos de sucesión en nuestro país, no lo hacen desde este enfoque sino indirectamente, ya que focalizan en otros problemas e interrogantes. Por un lado, centrada principalmente en la segunda mitad del siglo XIX e inicios del XX, Blanca Zeberio, analiza las redefiniciones jurídicas relativas a la familia, la propiedad y la herencia, a partir de las discusiones en torno a las posturas y tesis de jurisprudencia, ${ }^{10}$ durante el periodo de codificación. Encuentra en ellas que el creciente interés por redefinir los lugares de las mujeres casadas y los hijos en el orden sucesorio desde la década de 1850 , se desarrollaban en consonancia con las preocupaciones también expresadas en las tesis sobre el carácter de la propiedad privada. Por otro lado, también Selva Senor ilumina algunas cuestiones respecto al lugar de las mujeres en la herencia considerado en las tesis de jurisprudencia de principios del siglo

9. Para un estado de la cuestión al respecto en nuestro país, ver VASSALLO, Jaqueline, “¿Es posible hacer historia del derecho desde una perspectiva de género?”, en CELTON, Dora; GHIRARDI, Mónica y CARBONETTI, Adrián (coordinadores) Poblaciones Históricas. Fuentes, métodos y líneas de investigación, Río de Janeiro, Brasil, 2009, pp. 427-437.

10. ZEBERIO, Blanca, "Los hombres y las cosas.

Cambios y continuidades en los derechos de propiedad", en Quinto Sol, Santa Rosa, 2005/ 2006, No 9-10, pp. 151-183 Disponible en: <http://www.scielo.org.ar/pdf/ quisol/n9-10/n9-10a06.pdf >; ZEBERIO, Blanca, "Los derechos civiles en la transición...”, Op. Cit. 
$\mathrm{XIX}^{11}$ que demuestran una preocupación por parte de los juristas, por ubicarlas en función de su rol de madres y esposas. Por último, más específicamente desde la historia de las mujeres y los estudios de género, Dora Barrancos señala que la obturación de los derechos civiles tuvo como consecuencia la inferiorización de la condición jurídica de las mujeres, sosteniendo la agudización de la subordinación legal femenina en el tránsito del siglo XVIII al XIX. ${ }^{12}$

Teniendo en cuenta tales aportes y como punto de partida de nuestra investigación sobre las redefiniciones jurídicas del lugar de las mujeres en las dinámicas de sucesión, focalizaremos en este trabajo específicamente sobre las disposiciones del Código Civil de 1869. Ello nos permitirá a su vez reflexionar acerca del proyecto político y social que dicho Código encarna dentro del proceso de formación del Estado. En esta oportunidad, nuestro análisis permanece en el orden de las reglamentaciones, en un intento por esbozar cambios y continuidades en las concepciones existentes acerca del lugar de las mujeres en materia hereditaria en la etapa de codificación. Es por ello, que si bien buscamos analizar las disposiciones del Código Civil de 1869,

11. Principalmente que discuten el lugar de la mujeres en la herencia en caso de una sucesión ab intestato. Legalmente la esposa no era considerada heredera forzosa en caso de fallecimiento de su marido sin haber testado. SENOR, Selva, "La institución de herederos en la sucesión ab-intestato: Transformaciones en la concepción de familia y herencia. Buenos Aires durante la primera mitad del Siglo XIX", Quinto Sol, Santa Rosa, 2004, No 8, pp. 73-87.

12. BARRANCOS, Dora, "Inferioridad jurídica y encierro doméstico" en GIL LOZANO, Fernanda, PITA, Valeria y INI, María Gabriela (directoras) Historia de las mujeres en Argentina. Colonia y siglo XIX, Taurus, T. I, Buenos Aires, 2000. dedicaremos un breve apartado a la normativa previa existente.

\section{Mujeres y herencia en la legislación castellana}

Durante el período colonial y hasta la sanción de los códigos civiles (que tuvieron lugar entre el último cuarto del siglo XIX y primeras décadas del $\mathrm{XX})^{13}$, el ordenamiento social en Hispanoamérica estuvo regido por el derecho castellano. Si bien se puede hablar de la existencia del derecho indiano, el mismo no implicó una estructuración jurídica novedosa sino que, fue un intento de trasladar el orden jurídico de la península, (particularmente Castilla) al Nuevo Mundo. ${ }^{14}$ De esta forma, la legislación que regulaba las prácticas hereditarias durante el período colonial se basaba en el sistema castellano. Las Leyes de Toro (1505), eran las principales, así como, también la sexta partida de las Siete redactadas por Alfonso X, y el Fuero Real además de las diferentes cédulas y Pragmáticas que finalmente fueron sistematizadas en La Nueva Recopilación (1567) y, más tarde, la Novísima Recopilación (1805).

En relación al lugar destinado a las mujeres dentro de la dinámica de sucesión,

13. VASALLO, Jaqueline, "La construcción de la feminidad y la masculinidad en la doctrina jurídica y su impacto en la legislación argentina del siglo XX" en GHIRARDI, Mónica (compiladora) Familias Iberoamericanas ayer y hoy. Una mirada interdisciplinaria, ALAP Editor, Córdoba, 2008.

14. Para un recorrido detallado de las características del derecho castellano en América, ver BRAGONI, Beatriz, "El derecho castellano dentro del sistema jurídico indiano", en Anuario Mexicano de Historia del Derecho, 1998, No 10, pp. 89-105. 
este derecho les otorgaba el acceso a los bienes patrimoniales y también la capacidad de transmitirlos, ya que el sistema hereditario era bilateral, es decir que los hijos podían heredar tanto del padre como de la madre. Por lo tanto, ellas podían acceder a los bienes patrimoniales en primer lugar, como miembro integrante de la familia, en calidad de herederas, bienes de los cuales disponían a su voluntad como "parafernales", es decir, aquellos adquiridos antes del matrimonio. Luego de celebrado éste, las mujeres podrían aumentar su caudal, a través de aquellos bienes adquiridos durante el mismo, es decir, los bienes gananciales, de los cuales les correspondían la mitad a la muerte de su cónyuge. Por último, el marco legal brindaba a las mujeres elementos para su protección económica en caso de muerte o alejamiento del cónyuge: la dote y las arras. En el primer caso, era otorgado por la familia de la novia y, si bien su objetivo era darle un valor dentro del mercado matrimonial permitiéndole casarse con alguien del mismo sector social o superior, también era un elemento de protección económica en caso que el matrimonio cayera en desgracia ya que en este caso, volvía a las mujeres. ${ }^{15}$ Las arras eran añadidas por el novio en calidad de regalo y también pertenecían a las mujeres. Tales eran los bienes que, luego de su muerte, ellas podían transmitir, por lo tanto, vemos cómo su lugar dentro del grupo familiar, les permitía mantener su personalidad legal y económica, como herederas y otorgantes de herencia.

Estos elementos de protección y resguardo

15. SIEGRIST, Nora, "Ciudad y campaña bonaerense. Bienes tangibles e intangibles en la cesión de dotes y patrimonios. Siglos XVII-XIX" en SIEGRIST, Nora y ZAPICO, Hilda (coordinadoras) Familia, descendencia y patrimonio en España e Hispanoamérica siglos XVI y XIX, Eudém, Mar del Plata, 2010. hacia las mujeres convivían con otros de carácter restrictivo, producto de una concepción que las sometía a la tutela masculina, y que era resultado, además, de unas normas legales que tenían como objetivo final no la protección de las mujeres sino la de la institución familiar. La tutela correspondía, en primer lugar, al padre y luego al marido quién disponía de la libre administración de los bienes gananciales (sin limitaciones durante la unión, aunque debía restituir la mitad si ésta se disolvía), la dote y las arras (si bien también debía restituirla ya que en ambos casos no eran enajenables), así como de los parafernales en caso que las mujeres lo hubieran facultado voluntariamente para ello. El hombre era también el tutor natural de los hijos, luego de cumplidos sus tres años de edad.

De esta manera, podemos decir que la capacidad jurídica de las mujeres solteras (sometidas a la tutoría del padre), así como, de las casadas (a la del marido) era relativa. Como caso excepcional a esta situación, varios autores han destacado el carácter de autonomía plena del que gozaban las mujeres en estado de viudez que les permitía (siempre y cuando no mediara acusación de adulterio) conservar el disfrute de su dote y arras además de sus bienes parafernales y su cuota dentro de los gananciales, pudiendo además ejercer la tutoría de sus hijos menores después del periodo de lactancia, administrando sus bienes, siempre y cuando no contrajesen nuevo matrimonio, ya que se consideraba que las mujeres sujetas al amor conyugal perdían el buen juicio a diferencia del hombre que tendría un ánimo más firme y constante. Protección y restricción, capacidad jurídica relativa, asignación de roles, lugares y espacios determinados para hombres y mujeres eran 
algunos de los postulados que podemos vislumbrar a través de las disposiciones del derecho castellano y que emanaban de los códigos medievales y renacentistas tempranos, ${ }^{16}$ elaborados por filósofos, teólogos y moralistas cuyas concepciones se basaban por un lado, en supuestos de las tradiciones grecolatina y judeocristiana que otorgaban un lugar secundario a las mujeres ${ }^{17}$ así como en los textos bíblicos.

Aristóteles consideraba que los cuerpos masculino y femenino eran adaptados, específicamente a sus roles y cualidades particulares y naturales, dividiendo los espacios en esferas pública y doméstica. Es preciso aclarar en este caso, que a diferencia de las explicaciones modernas, no estamos frente a un intento por justificar los roles sociales en base a la naturaleza, sino que las propias categorías sociales son naturales y se encuentran en el mismo nivel explicativo que los hechos físicos o biológicos. En la antigüedad, el cuerpo de la mujer, no era una categoría ontológica, sino una versión menos perfecta del cuerpo masculino, ordenándose ambos cuerpos jerárquicamente como versiones de uno solo que se complementaban. ${ }^{18}$

Por otro lado, en las sagradas escrituras también se establecía una jerarquía que provenía de la prioridad cronológica de la creación del

16. LAVRIN, Asunción, "La mujer en la sociedad colonial hispanoamericana”, en BETHELL, Leslie, Historia de América Latina, T. IV, Editorial Crítica, Barcelona, 1990.

17. DUBY, George y PERROT, Michelle, Historia de las mujeres en Occidente, "Presentación”, T. 1, Taurus, Madrid, 1991, pp. 7-27.

18. Respecto a esta tradición ver: LAQUEUR, Thomas, La construcción social del sexo. Cuerpo y género desde los griegos hasta Freud, Feminismos, Madrid, 1994. varón por parte de Dios y la posterior creación de la mujeres a partir de la sustancia del varón y con el objeto de ser su compañera, así como, de la prioridad cronológica de la mujeres en el pecado original. ${ }^{19}$ De esta forma, así como el hombre era gloria de Dios, la mujer lo era del varón mientras que la asociación a la figura de Eva, las suponía portadoras de peligrosidad, así como de cualidades "carnales", "irracionales", "frágiles", "engañosas", "impuras" y al mismo tiempo, como madres. Posteriormente, a partir del culto mariano, ellas también serían símbolo de cuidado y amor al igual que la madre virgen de Dios, dando origen a una concepción ambivalente de su condición. ${ }^{20}$

Estas son algunas de las representaciones ${ }^{21}$ que se encontraban presentes en los postulados del derecho castellano y que éste reproducía asignando roles, lugares, capacidades y

19. BAÑARES, Juan Ignacio, "La mujeres en el ordenamiento canónico medieval (S. XII-XV)", en Anuario Filosófico, Universidad de Navarra, Pamplona, 1993, V.XXVI, No 3, pp. 559-572.

20. FOGELMAN, Patricia, "El culto mariano y las representaciones de lo femenino Recorrido historiográfico y nuevas perspectivas de análisis", en $\mathrm{La}$ Aljaba, Luján, 2006, V. X, pp. 175-188.

21. Utilizamos este término en el sentido de Chartier en el cual "no hay práctica ni estructura que no sea producida por las representaciones, contradictoras y enfrentadas por las cuales los individuos y los grupos dan sentido al mundo que les es propio". CHARTIER, Roger, El mundo como representación. Historia cultural: entre práctica y representación, Gedisa, Barcelona, 1995. p. 49. En este sentido, seguimos al autor cuando menciona que "la herencia de algunos trabajos de Mauss y Durkheim son fundamentales, particularmente la idea según la cual las representaciones colectivas incorporan en los individuos, de una manera social, las determinaciones del mundo social, y como se transforman en esquemas de percepción, de representación, de clasificación, las estructuras mismas de la sociedad". AYALA, Elisa, "Las ciencias sociales y la historia: una entrevista con Roger Chartier", en Takwá, Guadalajara, 2006, № 9, p. 169. 
características diferentes a hombres y mujeres operando desde lo ideológico y cultural. Sin tenerlas en cuenta, no podemos comprender completamente el lugar que la normativa les destinaba a las mujeres en dicho contexto.

\section{Mujeres y herencia en el Código Civil}

Era deber del nuevo orden, romper aquella trama de la sociedad colonial reemplazando el viejo sistema normativo por una codificación moderna elaborada al amparo de las experiencias de codificación en Europa y América Latina. Para ello, el Código Civil de Vélez Sarsfield (elaborado entre 1865 y 1869 a solicitud del Ejecutivo y promulgado en 1871) retomó las discusiones de los jurisconsultos del XIX, así como, los principios y reglamentaciones del Código napoleónico (1804) de la tradición liberal hispanoamericana inaugurada con el borrador del Código Civil español (1851), del Código chileno de Andrés Bello (1855) y del uruguayo de Eduardo Acevedo (1868) ya promulgados. Además del esbozo del brasileño de Freitas. ${ }^{22}$

El Código Civil tenía por objetivo, regular gran parte de la conducta privada de los habitantes y según Barrancos, sus efectos en relación a las mujeres fueron culminantes no sólo porque agravó la inferioridad femenina sino por su potencia instituyente. ${ }^{23}$

22. ZEBERIO, Blanca, "Disputar el patrimonio. Herencia, justicia y conflictos familiares en la Pampa Argentina (1880-1940)", en Anuario IEHS, Tandil, 2001, No 16, p. 367.

23. BARRANCOS, Dora, Mujeres en la sociedad argentina. Una bistoria de cinco siglos, Sudamericana, Buenos Aires, 2007, p. 101.
Específicamente en relación a la herencia y el manejo del patrimonio, las mujeres casadas se encontraban bajo la tutela del marido, situación que no les permitía ser administradoras de sus bienes ${ }^{24}$ y como tales no podían aceptar ni repudiar la herencia recibida sino con licencia de sus esposos y en su defecto la de un juez (en estos casos la voluntad del juez no debía contradecir a la del marido). ${ }^{25}$ En este sentido, incluso su calidad de herederas, como hijas y parte de un grupo familiar, se veía condicionada por su capacidad jurídica relativa al encontrarse bajo unión matrimonial. Dentro de estos términos, era el marido o el juez quien se veía habilitado para pedir o aceptar partición en nombre de su esposa si bien ella también podía hacerlo pero, únicamente, con previa autorización del cónyuge. ${ }^{26}$ De igual manera, las mujeres casadas sólo podían ser albaceas con licencia del marido ${ }^{27}$ y no podían desempeñarse como testigos.

Las mujeres viudas eran la excepción a esta regla ya que gozaban de capacidad jurídica plena siempre y cuando permaneciesen en ese estado pudiendo administrar libremente sus bienes, así como, ser tutora de sus nietos. En relación a la tutoría, el Código Civil expresaba que las mujeres, salvo las abuelas que hubieran permanecido en estado de viudez, no podían ser tutoras, declarándolas incapaces al igual que los menores de edad, los mudos, dementes, extranjeros, los carentes de oficio o modo de vida conocido, entre otros. ${ }^{28}$

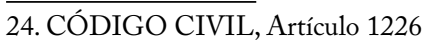

25. CÓDIGO CIVIL, Artículo 3334

26. CÓDIGO CIVIL, Artículo 3454 .

27. CÓDIGO CIVIL, Artículo 3847.

28. CÓDIGO CIVIL, Artículo 398. 
$\mathrm{Si}$ nos detenemos ahora en el principio de divisibilidad de la herencia, vemos como se mantuvo el reparto igualitario de bienes entre herederos forzosos pero se introdujeron algunos cambios significativos en relación al cónyuge ya que era considerado por la nueva legislación también como heredero más allá de los bienes gananciales que le correspondían. De esta forma, viudo o viuda e hijos legítimos recibían en la sucesión la misma parte ${ }^{29}$ (es decir, la quinta parte de los bienes del difunto). En caso que hayan quedado en compañía del viudo o viuda los ascendientes del difunto y no sus hijos legítimos, el cónyuge sobreviviente recibiría además de la mitad de los bienes gananciales, un cuarto de los bienes propios del difunto. ${ }^{30}$ Por último, sino quedasen ascendientes ni descendientes, los cónyuges se heredaban recíprocamente excluyendo a los parientes colaterales. ${ }^{31} \mathrm{La}$ viuda o viudo, también tendría derecho de habitación de forma vitalicia y gratuita, siempre y cuando no volviera a contraer nupcias. ${ }^{32}$ De esta forma, la posición del cónyuge era elevada junto con los padres en la segunda línea de sucesión.

En síntesis, la situación de las mujeres dentro de la dinámica hereditaria que el Código Civil dispuso, mantuvo su condición de herederas como miembros de la familia de quien fallecía, así como la propiedad sobre sus bienes y gananciales e incluso ascendía su posición como receptora de herencia en su calidad de cónyuge. Sin embargo, la propiedad efectiva sobre estos bienes que podía heredar y transmitir así como tales acciones permanecían

29. CÓDIGO CIVIL, Articulo 3570.

30. CÓDIGO CIVIL, Artículo 3571.

31. CÓDIGO CIVIL, Artículo 3572

32. CÓDIGO CIVIL, Artículo 3573. (en continuidad con el Derecho castellanoindiano) condicionadas por la tutela del marido, y sujetas por lo tanto a su estado civil. En referencia a las modificaciones introducidas en relación a la legislación previa, es decir, la inclusión del cónyuge en la línea de sucesión inmediata, creemos que más que considerar esta nueva situación como una concesión o mejora hacia el posicionamiento de las esposas, tal medida debe verse como parte del afianzamiento del modelo de familia legítima originada en el matrimonio, como transmisora de la propiedad privada. Podemos decir, que específicamente en materia hereditaria, si bien se introdujeron algunas modificaciones en relación a la legislación colonial, se mantuvo el principio castellano de división igualitaria de bienes así como la situación jurídica de las mujeres casadas.

Sin embargo, la continuidad de estos postulados dentro de un sistema normativo distinto, que se erigía como parte del proceso de construcción del Estado en el intento por centralizar su poder, en un contexto donde se habían producido también transformaciones socioeconómicas importantes en relación al periodo colonial, no implicaba la permanencia intacta de los mismos, sino que en este nuevo sistema, los mismos postulados adquirían sentidos distintos.

Al respecto, los avances realizados por Blanca Zeberio nos permiten iluminar estas cuestiones. A partir del análisis de las discusiones y debates de los jurisconsultos durante la segunda mitad del siglo XIX y que serían expresados en el Código Civil, (hecho que no debe extrañar ya que quienes formaban parte de tales discusiones fueron los dirigentes que participaron de la construcción político 
institucional) la autora demuestra como el creciente interés por redefinir el lugar de las mujeres casadas y los hijos en el orden sucesorio desde la década de 1850 se desarrollaba en sincronía con las preocupaciones, también expresadas en las tesis durante esos años, sobre el carácter de la propiedad privada. Las tensiones se situaban en torno a la relación dominio-propiedad que reflejaban las diversas formas de propiedad que convivían en el Rio de la Plata desde tiempos coloniales fundada en la figura legal de la posesión y en concepciones que propugnaban el fundamento de la misma en las leyes de Estado. Finalmente, el Código se alejó de aquellas posturas que establecían como sinónimos dominio y propiedad definiendo algunas prácticas de apropiación como hurto. Por su parte, en relación a la transmisión de la misma, la autora sostiene la existencia de un acuerdo estable en torno al establecimiento de un régimen legal de sucesión igualitario tendiente a evitar la inmovilidad de la propiedad, a la vez que favorecía su circulación de acuerdo a principios liberales, lo que se reflejaba además en la extinción de mayorazgos, censos enfitéuticos y capellanías así como en la limitación de los arrendamientos. Por otro lado, las discusiones giraban en torno al lugar de los hijos legítimos, ilegítimos y esposa en el orden sucesorio. Los hijos legítimos, heredaban sin distinción de sexo y de pleno derecho los bienes paternos mientras que la situación de los hijos nacidos fuera del matrimonio era menos igualitaria y presentaba una gradación de situaciones: los hijos naturales si bien excluían a los colaterales en el derecho a la herencia, tenían derecho a un cuarto de los bienes que le correspondían al hijo legítimo De la misma forma, las mujeres amancebadas y los hijos ilegítimos fueron excluidos del derecho a la herencia, manteniéndose incluso la denominación de hijos sacrílegos, a los que se privaba de toda pertenencia social y familiar. ${ }^{33}$ De esta forma, el Código buscaba reforzar la familia legítimamente constituida como transmisora de la propiedad. Asimismo, el interés por defender la familia legítima y su relación con la transmisión de la propiedad se reflejaba también en la preocupación expresada en las disposiciones por la contracción de nuevas nupcias por parte de la viuda, caso en el cual perderían no sólo la tutoría de sus hijos sino también del derecho de habitación.

De esta forma, insertando nuestro análisis sobre el lugar de las mujeres en la dinámica hereditaria dentro de las concepciones más amplias que el Código encarnaba en relación a la propiedad y la transmisión patrimonial estudiadas por esta autora, podemos ver como elementos del derecho anterior son mantenidos en tanto eran operativos en el nuevo orden. ${ }^{34}$

Por lo tanto, tal como plantea Blanca Zeberio, si bien las construcciones jurídicas del Código marcaban un cambio en la concepción del derecho introduciendo (en consonancia con las transformaciones económicas y políticas de la época) concepciones basadas en la libertad del individuo y la igualdad de derechos, en relación a la situación de las mujeres este sistema jurídico mantuvo la incapacidad jurídica de las esposas en pos de fortalecer a la familia legítima patriarcal como transmisora de propiedad.

33. ZEBERIO, Blanca, "Los hombres y las cosas..." Op. Cit.

34. Sobre el mantenimiento de elementos residuales de la legislación anterior en los nuevos códigos, más allá de los aspectos sucesorios ver: NARI, Marcela, Políticas de maternidad y maternalismo político, Buenos Aires, 18901940, Biblos, Buenos Aires, 2004. 
Esta situación es observada no sólo en los aspectos sucesorios sino que Verónica Giordano lo analiza también en el proceso de construcción de ciudadanía, en el cual la democracia moderna se ha erigido sobre la contradicción entre una instancia de igualdad formal y una instancia real en la que son manifiestas las diferencias y las exclusiones, ${ }^{35}$ planteando la validez de la paradoja señalada por Joan Scott para el caso francés, donde las formulaciones que intentaron incluir a las mujeres en un régimen de igualdad, al mismo tiempo que reivindicaron la universalidad de la igualdad como valor asociado a la condición humana, justificaron dicha inclusión a partir de un criterio particular, la diferencia sexual, esto es la identificación de las mujeres como madres y esposas. ${ }^{36}$

En relación a este aspecto, resultan pertinentes los elementos que introducen las feministas ${ }^{37}$ que desarrollan cómo la separación entre lo público y lo privado que plantea la teoría liberal, ha sido operativa para ocultar dentro de un orden aparentemente universal, igualitario e individualista, la sujeción de las mujeres.

Consideramos que adoptar una mirada de

35. GIORDANO, Verónica, Ciudadanas incapaces. La construcción de los derechos civiles de las mujeres en Argentina, Brasil, Chile y Uruguay en el siglo XX, Editorial Teseo, Buenos Aires, 2012, p.31.

36. Ídem, p. 36

37. PATEMAN, Carole, "Críticas feministas a la dicotomía público/privado" en CASTELLS, Carme (compiladora) Perspectivas feministas en teoría politica, Paidós, Madrid, 1996. FRASER, Nancy, "Repensando la esfera pública: una contribución a la crítica de la democracia actualmente existente", en Debate feminista, México, 1993, № 4. género, a la hora de abordar estos discursos resulta fructífero para identificar en qué contexto y de qué manera configuran, instituyen y reproducen estereotipos que se presentan como "objetivos", "naturalizados" y “atemporales".

Hasta aquí, hemos avanzado en nuestro estudio sobre el lugar de las mujeres en las dinámicas de sucesión, focalizando en las disposiciones del Código Civil con el objetivo de rastrear cambios y continuidades con la legislación previa en pos de historizarlas. Sin embargo, sabemos que el derecho no es la única dimensión que influirá en la construcción de los modelos de familia, así como del lugar que ocupan sus miembros, y los roles que deben desempeñar, sino que este se articula históricamente con otros discursos que, además de la religión y la moral, cobrarán preponderancia con el correr de los años como el biomédico, de la economía doméstica, ${ }^{38}$ educacional.

Por otro lado, debemos destacar que la historia de las mujeres en Argentina, ha desarrollado prolíficos trabajos que se han centrado en recuperar la agencia de éstas en distintos procesos históricos a partir del análisis de la interacción entre normas y prácticas, demostrandocómolas mujeres han desarrollado estrategias de acción tanto individuales como colectivas que les permitieron no solamente sortear las imposiciones del orden social sino generar resistencias y resignificaciones que cuestionaron los roles y espacios asignados a partir de desigualdades sexuales. ${ }^{39}$

38. NARI, Marcela, Políticas de maternidad..., Op. Cit.

39. PALERMO, Silvana, “Género y ciudadanía política: algunos apuntes en la agenda de investigación", en PolHis. Boletín Bibliográfico Electrónico del Programa de 
En el caso de las dinámicas hereditarias, en algunos de nuestros trabajos hemos comenzado a explorar desde un enfoque atento a las relaciones entre norma y práctica, basado en el análisis de sucesiones y fuentes judiciales, el lugar de las mujeres campesinas del periodo tardo colonial bonaerense en los que vimos cómo ellas participaban activamente resignificando las diferencias genéricas que les otorgaban un lugar subordinado al hombre en la sociedad, desempeñando papeles más activos de los que los ideales les asignaban a través de la utilización de los intersticios legales disponibles para reclamar por sus derechos así como para generar estrategias de solidaridad con otras mujeres. ${ }^{40}$ En este sentido, orientamos la continuidad de nuestra investigación insertando la problemática de la herencia dentro de la trama de relaciones sociales que sustentaba la vida cotidiana y la reproducción familiar, procurando profundizar el estudio de las normas y prácticas de transmisión de patrimonio.

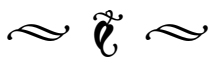

Buenos Aires de Historia Politica, Mar del Plata, 2011, primer semestre, No 7; PITA, Valeria, La casa de las locas. Una historia social del Hospital de Mujeres Dementes. Buenos Aires, 1852-1890, Prohistoria Ediciones, Rosario, 2012.

40. CORONELLO, Romina, "Herencia y familias campesinas: el lugar de la mujer rioplatense tardo colonial", en Zona Franca, Centro de estudios interdisciplinarios sobre las mujeres, UNR, Rosario, 2010, Año XVIII, No 19. 